\title{
A post-take ward round
}

\author{
Patrick Vallance
}

J R Soc Med 2005;98:191-192

It's 2005 and I work as a clinical academic at a large teaching hospital in London. My time is divided between research, teaching and clinical practice, with a fair whack of administration. General medicine, which once vied with general surgery for top position in a teaching hospital hierarchy, has like its cutting cousin slipped down the priority list and is being usurped by specialist interests and immediate triage of patients into organ-based silos. Nonetheless, the reality is that patients present with undiagnosed illness and general medicine remains in the front line.

I rather like general medicine-the challenge of diagnosis and the need to think broadly about what will make the patient better and get him or her back home. Our round starts on the coronary care unit and the group consists of a night team, a day team, some of yesterday's team and a cardiologist. In the past two years the need to meet a new working-time directive from the European Union means that a form of shift work has arrived. The number of doctors on the round has increased but the chances of any one doctor knowing the full course of the patient from admission to post-take round is virtually nil. On the other hand, the junior doctors come into contact with many different consultants and the consultants get to know most of the junior staff. An advance? Not in terms of continuity of care or team spirit, but perhaps as we get used to working in shifts these problems will resolve.

The first patient has had chest pain - currently labelled by the catch-all of 'acute coronary syndrome, ACS'. No changes on the electrocardiogram but troponin is raised. Closer questioning reveals that he had a chest infection for a week or so beforehand. Might it be that the chest infection triggered a coronary event ${ }^{1}$ or could the troponin be a falsepositive secondary to infection ${ }^{2}$ A lively discussion ensues, covering disease mechanisms, the nature of plaque rupture and the criteria that make a reliable diagnostic test. The discussion ends, inevitably, in the patient being booked for angiography. The round of the coronary care unit is interesting and we can try to apply evidence-based treatments. The evidence base in cardiovascular medicine

Patrick Vallance, MD FMedSci, is Head of the Division of Medicine at University College London. His principal research interest over the past decade has been the role of vascular endothelium in the regulation of human vascular tone and blood-vessel/vessel-wall interactions.

Divison of Medicine, Rayne Institute, 5 University Street, London WC1E 6JF, UK E-mail: patrick.vallance@ucl.ac.uk is strong and often generally applicable to the population of inpatients. The hidden pitfall lies in relative versus absolute risk. Everything we discuss seems to reduce risk by 30\%, but of course the key question is ' $30 \%$ of what?'. Our inability to calculate accurately the absolute risk in many of the patients means that we cannot really estimate the incremental benefit of each new treatment we propose. Better risk prediction, possibly incorporating genetic risk, seems like a worthwhile goal. We also leave with an uneasy feeling that we haven't really prevented cardiovascular events with the new treatments we have started but merely delayed them, and we don't know by how long.

We move out of the coronary care unit and start on our trail around ten wards. First stop is a young man who is a drug addict. He feels unwell and has a pyrexia. Our thoughts immediately turn to bacterial endocarditis - a disease that most hospital doctors consider rare but one which in central London, close to King's Cross, we see almost every week. Intravenous access is difficult, and once the central line is in place the patient is likely to disappear regularly from the ward for a quick fix. We can treat the infection but we can do nothing to solve the underlying problem. There's a lot to teach on with this patient and the students have many questions. We raise again the issue of whether there is a need for someone (us?) to write a handbook of urban medicine.

Onwards and upwards. We walk to the top of this block of the hospital. We walk because we want to. Because we believe that regular exercise is good for us. Is there evidence that walking up five flights of stairs is good for us - no, but we choose to do it anyway. At the top we get an invigorating view of our new hospital, a $£ 422$ million investment, much of it funded under the Private Finance Initiative (PFI). The very thought of working in a state-ofthe-art, new, clean and single-site hospital is enough to keep us all enthused. And in true UCL tradition, one of the most fierce critics of PFI is a professor at UCL. ${ }^{3}$ Will we be forgiven for taking out such a big mortgage? Reader in 2055, you will know the answer. At the moment, on this ward round stretching across ten wards and three separate sites, in deteriorating buildings, the answer seems like a resounding yes.

We see a patient who was admitted with headache. After various tests she was diagnosed as having migraine and was given a triptan. The headache resolved. This is a clear therapeutic success. But if only we could search the 


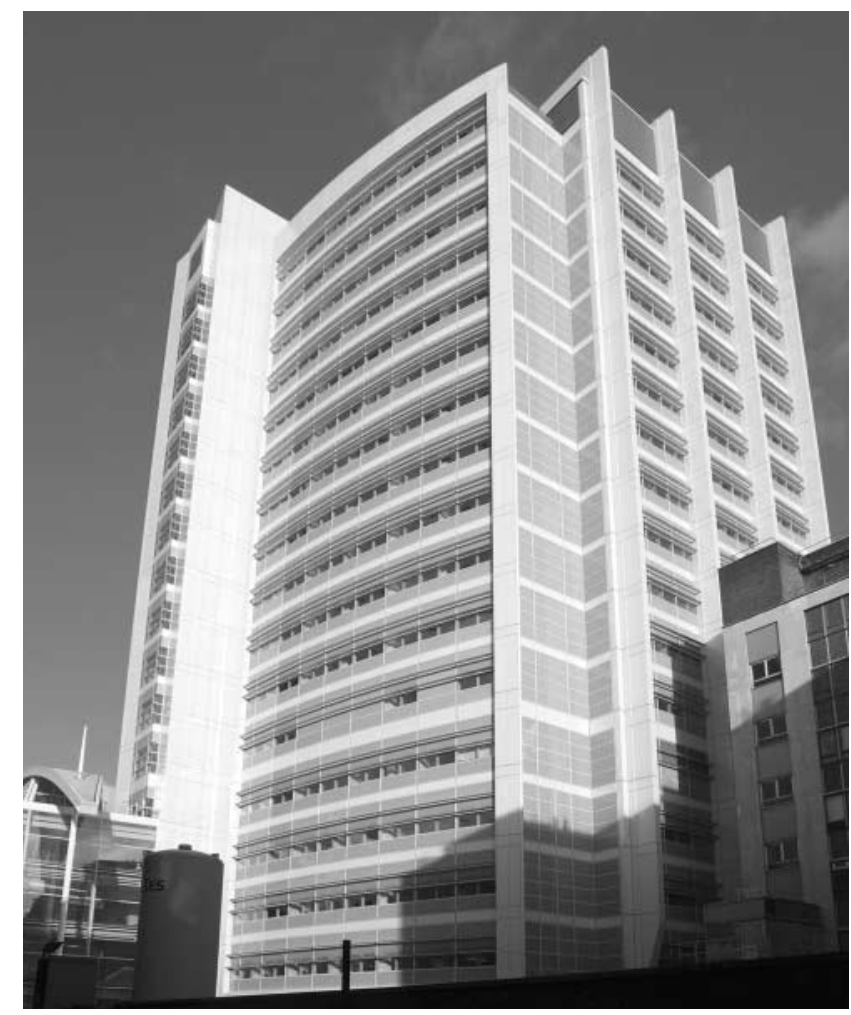

published work on the ward round we would find that there was a $40 \%$ likelihood of this happening by chance, that aspirin plus metoclopramide might well have been just as good, and that intravenous aspirin might have given the same result, possibly with fewer side-effects..$^{4-6}$ Nonetheless we get the positive reinforcement of having achieved a therapeutic success, even if in reality it owed as much to that mysterious entity the placebo effect as to magic-bullet therapeutics. In this behaviour - of believing that what we see is always attributable to the agent we administer - we are as guilty as the homeopathists. The differences are that sometimes our therapeutic agent does work and sometimes it causes harm.

Social problems loom. Here is an elderly woman with a left hemiparesis. Compare the medical inaction in response to this atherosclerotic complication with the excitement, intervention and kudos that surrounds the management of coronary artery disease. We are fortunate in that stroke is taken seriously here and most patients are transferred quickly to the acute brain injury service at Queen Square. Not so in many hospitals, and in this instance there were no beds on the unit. We check that aspirin has been prescribed and discuss physiotherapy, occupational therapy and the home situation. We anticipate a long stay, much of it not requiring any specific medical intervention, and a long complex discharge process. These problems require integrated teams and to some extent these have been lost. Reinvention of teams of doctors, nurses, therapists and social workers seems like an important task for general
A few wards later we reach our diagnostic challenge- - a man with a fever who works at night maintaining the Underground and frequently comes into contact with rats. The excitement of leptospirosis has spread contagiously through the ranks, taking firm hold of those closest to MRCP. Will it be, won't it be? Let's get the tests off, but since he had a week-long history of cough and shortness of breath perhaps we should treat him for an atypical pneumonia as well. Fortunately, a polymerase chain reaction test is available to aid more rapid diagnosis.

So back to the research laboratory. Here we are looking at a time-scale of months, not hours or days - the long slog of designing robust studies and interpreting results. The clinicians in the laboratory find the change of time-frame disconcerting. They miss the daily gratification of patient care, but ultimately some get hooked when they feel the excitement of being the first to know something new. Perhaps the work will eventually impact on our behaviour on the post-take ward round? To achieve this we will need to keep promoting clinical research, particularly experimental medicine. ${ }^{7}$ We have had worms (Caenorhabditis elegans), zebra fish and mice as model organisms and they remain immensely valuable, but, as Sidney Brenner has pointed out, we are now in an era when we have the genetic map and the techniques that make man a model organism for probing physiology and disease.

One of our new clinical research fellows was an outstanding student at UCL. He came back because he enjoyed being taught clinical medicine by research-active clinicians. Another example of why the idea that you should only do two out of teaching, research and clinical practice is wrong.

\section{REFERENCES}

1 Smeeth L, Thomas SL, Hall AJ, Hubbard R, Farrington P, Vallance P. Risk of myocardial infarction and stroke after infection or vaccination. $N$ Engl J Med 2004;351:2611-18

2 Ammann P, Pfisterer M, Fehr T, Rickli H. Raised cardiac troponins. BMJ 2004;328:1028-9

3 Pollock AM, Shaoul J, Vickers N. Private finance and 'value for money' in NHS hospitals: a policy in search of a rationale? BMJ 2002;324: 1205-9

4 Subcutaneous Sumatriptan International Study Group. Treatment of migraine attacks with sumatriptan. N Engl J Med 1991;325:316-21

5 Oral Sumatriptan and Aspirin plus Metoclopramide Study Group. A study to compare oral sumatriptan with oral aspirin plus oral metoclopramide in the treatment of migraine. Eur Neurol 1992;32:177-14

6 Diener HC. Efficacy and safety of intravenous acetylsalicylic acid lysinate compared to subcutaneous sumatriptan and parenteral placebo for the acute treatment of migraine. A double blind double-dummy randomized, multicenter, parallel group study. Cephalagia 1999; 19:581-8

7 Peters K. Exceptional matters. Lancet 2004;364:2142-51 